\title{
CARDIOSOUNDS: A PORTABLE SYSTEM TO SONIFY ECG RHYTHM DISTURBANCES IN REAL-TIME
}

\author{
Andrea Lorena Aldana Blanco ${ }^{1}$, Steffen Grautoff ${ }^{2}$, Thomas Hermann ${ }^{1}$ \\ ${ }^{1}$ Ambient Intelligence Group, CITEC, Bielefeld University, Bielefeld, Germany \\ ${ }^{2}$ Klinikum Herford, Emergency Department, Herford, Germany \\ aaldanablanco@techfak.uni-bielefeld.de
}

\begin{abstract}
This paper is a continuation and extension of our previous work [1]. CardioSounds is a portable system that allows users to measure and sonify their electrocardiogram signal in real-time. The ECG signal is acquired using the hardware platform BITalino and subsequently analyzed and sonified using a Raspberry Pi. Users can control basic features from the system (start recording, stop recording) using their smartphone. The system is meant to be used for diagnostic and monitoring of cardiac pathologies, providing users with the possibility to monitor a signal without occupying their visual attention. In this paper, we introduce a novel method, anticipatory mapping, to sonify rhythm disturbances such as Atrial Fibrillation, Atrial flutter and Ventricular Fibrillation. Anticipatory mapping enhances perception of rhythmic details without disrupting the direct perception of the actual heart beat rhythm. We test the method on selected pathological data involving three of the most known rhythm disturbances. A preliminary perception test to assess aesthetics of the sonifications and its possible use in medical scenarios shows that the anticipatory mapping method is regarded as informative discerning healthy and pathological states, however there is no agreement about a preferred sonification type.
\end{abstract}

\section{INTRODUCTION}

Sonification of biosignals in medical scenarios is a common method to monitor and detect abnormalities in specific signals. A well-known example are pulse oximeters, medical devices to monitor the oxygen saturation levels, that produce a short sound in synchronization with the pulse rate. The starting frequency of the sound is defined by each company that manufactures the devices [2], however, when the oxygen saturation level decrease, regardless of the manufacturer, a sound of a lower frequency is triggered, calling for the immediate attention of clinicians.

In current times, besides the well-known sound of the pulse oximeter, sonification research focuses on an extended set of biosignals, among which is the electrocardiogram (ECG). One of the reasons for this is that the human hearing system has a great capability to recognize changes and patterns in the signal, therefore sonification appears to be a powerful tool for diagnosis and monitoring of multivariate time series such as the ECG [3] [4]. In fact, some ECG monitors already include a sonification that triggers a

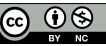

This work is licensed under Creative Commons Attribution Non Commercial 4.0 International License. The full terms of the License are available at http://creativecommons.org/licenses/by-nc/4.0 short duration sound whenever there is a heartbeat, providing information about the heart rate. However, there are still many other parameters in the ECG signal that can be sonified to present a more complete view of the current state of the heart. Another reason for using sonification, is that monitoring through sounds allows users to focus their visual sense on a primary task while being aware of the changes in the monitored signal [5], and this is an interesting approach in situations where users need to perform a task while tracking changes in a signal.

Based on the previously explained observations, we have identified two cases in which ECG sonification could be applied: (i) ECG auditory biofeedback when doing sports activities and (ii) auditory ECG monitoring to assist diagnosis during sport tests (e.g. Cardiac stress test). In the first case, sonification permits users to receive information about their heart rhythm when they are doing sport activities without blocking their visual sense. This is particularly important in activities where the users should focus their attention on the activity itself and avoid distractions from looking at a visual monitor (e.g. in jogging, biking). In the second case, clinicians can monitor the ECG signal from a patient while focusing their visual attention on other tasks such as examining the patient or looking at their clinical history.

In this paper, we first explain the ECG signal and particularly focus on the relevant background on arrhythmic patterns in ECG. We then introduce the CardioSounds system architecture which allows modular extension of other sonifications, recording of ECG data and interactive control of sonification parameters. We then introduce the anticipatory mapping method for sonifying arrhythmias as a concept and how to implement it in python/Supercollider. Subsequently we demonstrate the sonification at hand of selected healthy and pathologic ECG sonifications together with their clinical interpretation. Section 5 presents the results of the preliminary perception test where we evaluate the ability of anticipatory mapping to assist detection of changes in rhythmical organization. We therein also evaluate the participants' assessment of the aesthetic qualities and we consider compatibility with current clinical soundscapes by involving stakeholders from a German hospital. Discussion and conclusions summarize the paper.

\section{ECG AND ARRHYTHMIAS}

An electrocardiogram is a visual representation of the electrical activity of the heart measured as the time series of electric potential differences between two selected points on the skin (e.g. left arm and right foot). A standard clinical ECG usually has 12 leads using 10 electrodes, but details are not further relevant for the unfolding of the sonification presented here. Let's first understand a single 
lead, e.g. Lead I (difference between left and right arm).

In each cardiac cycle a number of reference points can be spotted in the signal. Marking the beginning of the cycle, there is the $\mathrm{P}$-wave, which represents regular atrial depolarization. Then, there is the so-called QRS complex representing ventricular activation; starting in the ventricular septum and finishing in the left ventricle. However, activation in the right and left ventricles happens almost simultaneously, for this reason the QRS complex is generally considered all together. Subsequently there is a time between depolarization and repolarization depicted by the ST segment and finally, there is the $\mathrm{T}$ wave that results from ventricular repolarization (see Fig. 1).

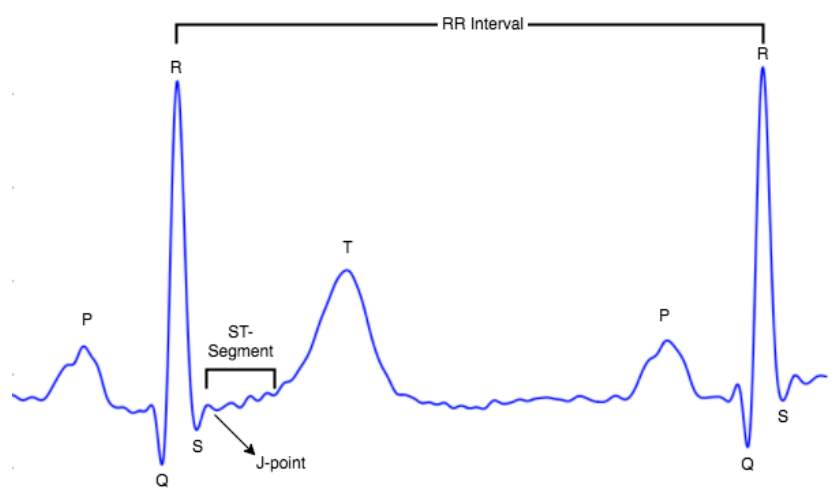

Figure 1: ECG standard reference points: P-wave, Q, R, S, J point, ST-segment, and T wave.

The reference points are the cues that clinicians use to determine if there are changes in specific parts of the heart, and if these changes correspond to a given pathology. For example, arrhythmias ${ }^{1}$ can be detected by looking at the variations in the duration of the RR interval (see Fig. 1) and the ECG waves' changes.

There are several types of arrhythmias that can be the result of impulse formation and/or conduction problems [6][7]. Nevertheless, not all rhythm disturbances are considered pathological. For instance, sinus arrhythmia, is a variation of the RR intervals that can result from respiration activity [8]. On the contrary, there are disturbances that are considered serious medical conditions which could lead to other diseases (e.g. strokes) and life-threatening arrhythmias that require immediate medical attention. Among these there are three examples of important arrhythmias:

1. Atrial fibrillation: Next to the usually benign conditions (premature atrial or ventricular beats and sinus arrhythmia), atrial fibrillation (AFib) is the most common arrhythmia. AFib can lead to a higher prevalence of blood clotting in the heart which increases the risk of stroke especially if the patient is comorbid. $^{2}$ Therefore, most patients who suffer from atrial fibrillation have to take anticoagulants (blood thinner) on a regular basis for prevention. In this condition the atrium is not able to contract efficiently. This decreases the heart's ability to pump which can lead to quicker exhaustion of the patient. AFib is a disease which is often caused by fibrosis of the atrium, but can be due to other causes, too. In AFib there are multiple irregular electrical

\footnotetext{
${ }^{1} \mathrm{~A}$ rhythm disturbance in the heart

${ }^{2}$ The patient has one or more additional diseases
}

impulses which lead to an irregular ventricular conduction. Instead of regular P-waves there are irregular fibrillatory (f) waves visible followed by irregular QRS complexes in AFib.

2. Atrial flutter: Atrial flutter (AFL) is also a common arrhythmia, but occurs less often than Afib. In AFL the rate of atrial beats is around 220 and 320 per minute. There is a delay of conducting these beats to the ventricles caused by the atrioventricular (AV) node. In AFL the AV node usually transfers the conduction in a regular pattern as in most cases it is $2: 1$ which is equivalent to blocking one after transferring one conduction. This leads to a ventricular rate of around 130 to 150 beats per minute. This rhythm disturbance also requires the intake of anticoagulants in most patients due to an increased risk of stroke. Additionally, it has an impact on the performance of the heart and the endurance of the patient.

3. Ventricular fibrillation: Ventricular fibrillation (VFib) is a life-threatening condition which requires immediate medical help for preventing sudden death. VFib is due to multiple chaotic electrical impulses in the ventricles which do not result in any regular contraction of the heart. In this condition the muscles of the heart are coming to a standstill and blood containing oxygen cannot circulate in the body. If VFib is present defibrillation (electric shock) is necessary without delay. This can immediately restore a regular heart rhythm such as a sinus rhythm. VFib can have multiple causes, but often occurs during the acute phase of myocardial infarction (MI) ${ }^{3}$. Due to lack of blood supply of the heart all kinds of rhythm disturbances can occur of which VFib is the most dangerous.

\section{THE CARDIOSOUNDS SYSTEM}

CardioSounds is meant to be used as a portable real-time ECG sonification system. Its structure can be decomposed into four modules: Input, processing, output and control, each with a hardware and software component. First, we explain the hardware components of the system:

(1) The input module uses a BITalino device ${ }^{4}$, a hardware platform that allows real-time data acquisition and wireless transmission of the recorded signals. (2) The processing module is done in a Raspberry Pi 3 Model B ${ }^{5}$, in which the signal is analyzed and sent to the audio engine. (3) The output module is a DIGITUS 7.1 USB Sound Box ${ }^{6}$ (portable sound card). Even though the Raspberry Pi has a headphone jack audio output, we use a small USB card in order to achieve better sound quality. Lastly, (4) the control module is a smartphone or tablet that allows the user to start and stop the signal acquisition and control basic settings such as the sonification type. A diagram of the system can be seen in Fig. 2.

On the following subsections, we explain how we process the signal in each module.

\footnotetext{
${ }^{3}$ Lack of oxygen supply in the heart due to a blocking of the coronaries, that in prolonged cases, could lead to death of the heart' tissue.

${ }^{4} \mathrm{http}: / / \mathrm{www}$.bitalino.com

${ }^{5}$ https://www.raspberrypi.org/products/raspberry-pi-3-model-b/

${ }^{6} \mathrm{https}: / / \mathrm{www}$.digitus.info
} 


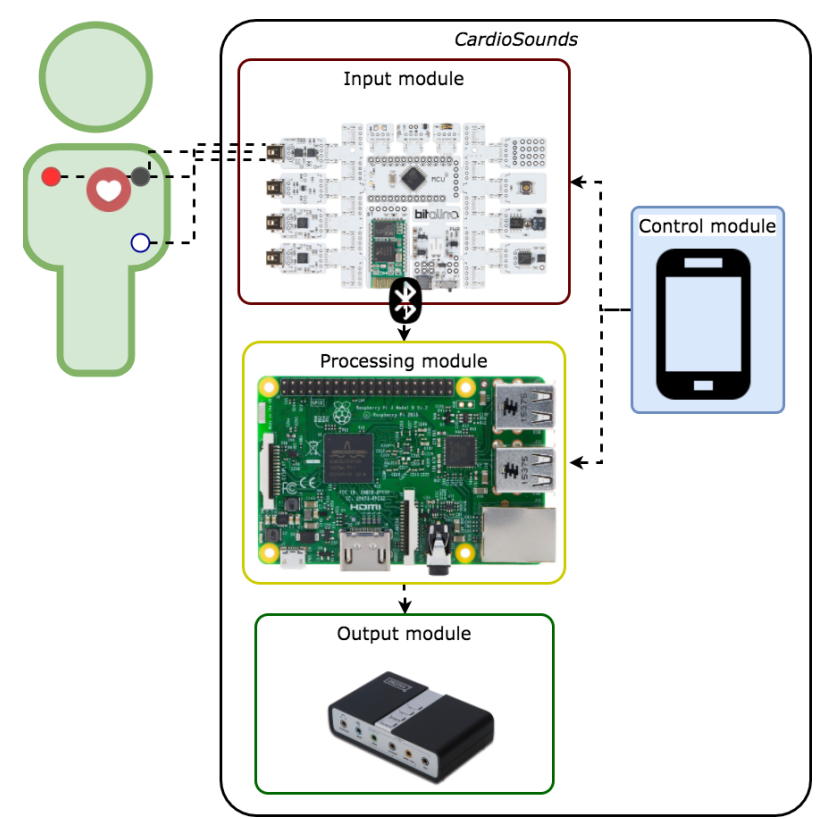

Figure 2: CardioSounds system architecture.

\subsection{Input module: Acquiring the ECG signal}

The input signal is recorded using BITalino. Currently we record one ECG lead (channel), however, it is also possible to extend to multivariate extensions by adding more ECG sensors to the system. The signal is sampled at $1000 \mathrm{~Hz}$.

\subsection{Processing module: $R$ peaks detection and $R R$ interval estimation}

Once the signal is acquired, we remove unwanted information before extracting the features of our interest. First, we remove the DC component and then apply a low-pass filter with a cutoff frequency of $70 \mathrm{~Hz}$. We do this in order to eliminate frequencies outside the accepted range for ECG diagnostics [9]. Additionally, we apply a band-stop filter to remove signal noise at $50 \mathrm{~Hz}$. Then, we perform the $\mathrm{R}$ peaks detection by applying the method proposed by Worrall et al. [10] within a time window of $200 \mathrm{~ms}$. The reason for this window size is that the typical QRS duration for a healthy adult with a heart rate of $60 \mathrm{bpm}$ is $100 \mathrm{~ms}$. Once at least two consecutive $\mathrm{R}$ peaks are detected, we can estimate the RR interval $\Delta t_{R R}$. While filtering can be done in real-time at sample rate and thus only causes a latency of few milliseconds, the RR-peak detection necessarily introduces a latency of $100 \mathrm{~ms}$ in the creation of events to be used for sonification. However, as monitoring of ECG is not such a time-critical task, and rhythms can actually only be judged from a longer series of heart beats, the latency is irrelevant.

\subsection{Output Module: Sonifying the signal}

When we have extracted the ECG features of interest, we define which parameters will be mapped to the sound features. Then, this information is sent using Open Sound Control (OSC) messages to the audio engine developed in Supercollider. In the output module, users can select the sonification type and the playback volume.
The modular construction of the system allows us to use this module with offline data too and control the temporal resolution of the sonification. The advantage in this case is that clinicians can inspect the ECG data by playing the sonification at faster or slower speed. Therefore, it is possible to do a quick scan of longer data files, or focus on a specific part of the data by playing it slowly.

\subsection{Control module: The CardioSounds app}

In order to control basic features of the system such as start and stop the signal acquisition, we developed a mobile application along with CardioSounds. Using the app we provide a control that contributes to the portability of the system. Also, we want to take advantage of devices that people and clinicians already use in their everyday life in order provide control of CardioSounds at lower costs.

The mobile app and the Raspberry Pi communicate through Open Sound Control (OSC) messages. Thus, the only condition is that they are in the same network. As the controls merely issue commands and parameter changes for sonification, latency is not a critical issue. Neither is bandwidth as currently only user actions such as start or stop are triggered. Later versions of Cardiosounds will enable to review ECG data on the smartphone, yet before data transmission can be implemented we need to make sure that necessary security measures and authentication is implemented. Hence so far, the visualization can only be done in a display connected to the Raspberry Pi.

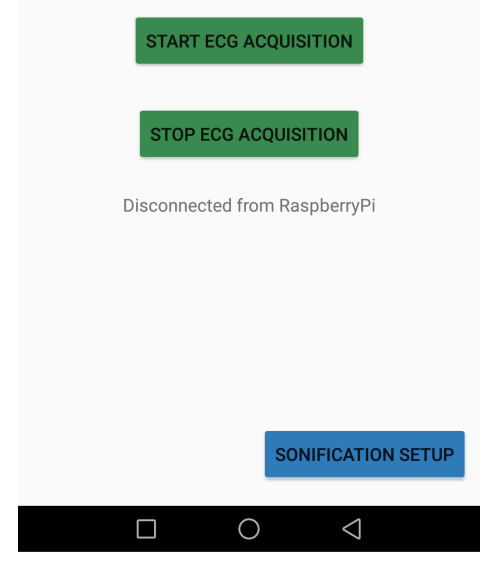

Figure 3: CardioSounds Mobile App.

\section{SONIFICATION DESIGN: ANTICIPATION MAPPING}

The human auditory system is already highly capable of detecting rhythms and irregularities [11]. The Just Noticeable Difference (JND) for detecting time displacement in a metrical sequence is about $10 \mathrm{~ms}$ when the duration between sound events is shorter than $250 \mathrm{~ms}$ or around $5 \%$ when the time between events is longer 
than this value [12]. Therein it might seem unnecessary to use any method at all (apart from playing sound events at the time of a heartbeat) and leave the assessment of rhythm to the user alone. So why use a method to enhance arrhythmia detection? First of all, if the interest is in subtle changes that fall below the JND, then rendering temporal changes more salient may help to assess them at greater accuracy. Secondly, in a situation of various sound sources competing for attention, perceivable yet small rhythmic variations may simply be overheard due to their limited saliency, particularly as habituation is at work to blend stationary sound streams more or less out. For these reasons, we here take a fresh look at ways to augment the standard pulse sonification but without interfering with the naturally audible rhythmic structure.

In essence the rhythm perception requires to be able to memorize a temporal pattern, to recognize parts of it in the stream of previously perceived events, and with increasing match between items of the memorized pattern and the heard pattern, an expectation for the timing of the subsequent events narrows down. If then the heard event doesn't agree with our expected beat onset, we witness a deviation which raises our attention as it contains more information (e.g. higher entropy) than the expected outcome. For the most simple type of rhythm a sequence of beats at equal time difference however, not much memory is required.

So, assuming that this anticipation and constant comparison of an anticipated onset with the observed onset happens already in the listener's auditory perception, would it be possible to connect to it and provide additional information about rhythmical deviations explicitly in the gap between the expected and the actual, whichever comes first?

This is exactly what we do by introducing the Anticipation Mapping, a parameter mapping sonification that adds a before-orafter shadow of the following event (here: heart beat) extending between the anticipated and actual time, whatever is first.

In case the anticipated beat comes first, we start a sound at that time to develop systematically in one attribute until the actual beat comes. If for instance, we choose pitch as the attribute, the resulting sound would up-chirp until the moment the sonification event of the real beat stops this before-shadowed component. In effect, we receive an explicit cue about the discrepancy in two variables: (i) an extra sound which temporally extends according to the difference between the anticipated and actual onset, and (ii) a pitch change which depends on the temporal difference. According to the choice of attributes and details of how to drive the parameter changes, we can fine tune the arrhythmia sonification to draw controlled attention to deviations larger than a defined threshold, for instance $5 \%$.

In case that the actual beat comes before the anticipated beat, we could for example play a sound that extends precisely to the moment of the anticipated beat - in this case a shadow of the sound. This will also mark specifically where the beat should have been according to expectations gained from the sequence of past beats.

In the following we will detail out different strategies to assess the anticipation and three different alternatives how to realize the anticipation mapping using concrete synths and mappings.

\subsection{Computation of the Anticipation signal}

\subsubsection{Constant Rate Anticipation}

The most straightforward method to compute the anticipation signal (i.e. the landing point in time at which the next beat would be expected) at hand of the observed previous data is by simply averaging their inter-beat intervals and adding the average to the last observed onset, in mathematical terms

$$
t_{i+1}=t_{i}+\frac{1}{M} \sum_{k=0}^{M-1}\left|t_{i-k}-t_{i-k-1}\right|=t_{i}+\frac{t_{i}-t_{i-M}}{M}
$$

This is a good estimate under the assumption of regular rhythm, i.e. that all heartbeat intervals should be equal. It would give poor anticipation potentials if the heart rhythm should contain some shuffling, i.e., every even heart beat is delayed from its regular rhythm. It also performs suboptimal if there is an acceleration or slowing down over time, which naturally occurs in human heart rhythms depending on cardiac stress. This motivates to consider Changing Tempo Anticipation which is introduced next.

\subsubsection{Changing Tempo Anticipation}

In this model, we compute the anticipation signal from applying a linear regression to the sequence of the past $M$ inter-heartbeat intervals, i.e., to the data set $\left\{k, t_{i+k}\right\}_{k=-M, \ldots,-1}$, fitting parameters $a, b$ of our model $h(k)=a \cdot k+b$. The expected time is then simply

$$
t_{i+1}=t_{i}+h(0) .
$$

Of course, more complex models are possible that consider rhythmical fine structure within the past beats, however these are not further considered at this stage of investigation. Figure 4 shows how the two forecast methods perform when $M=20$.

\subsection{Sonification designs}

We propose three sonification designs based on the anticipation signal computation: (i) Polarity-Pitch, (ii) ECGrains and (iii) Marimba. All methods intend to magnify time changes between actual and expected onsets by mapping the anticipation signal to a set of auditory parameters.

\subsubsection{Polarity-Pitch Sonification}

The Polarity-Pitch sonification intends to magnify the time difference between the actual and expected R peaks, specifically, when this difference falls below the just noticeable difference. In order to do so, when an R peak is found, we compare the time difference to the expected onset (forecast), and trigger a sound with a duration equal to the previously mentioned time difference. By doing this, we have already an idea of how arrhythmic the signal is, however, despite of our high capacity to discern rhythm variations, our auditory system could miss subtle variations and clues about how the RR interval is increasing or decreasing. For this reason, we also take into account the order in which the actual and expected onset take place, and map this parameter to pitch of the sound event. If the actual onset happens after the expected onset, we trigger a sound of a given frequency, and for the opposite scenario, we trigger a sound an octave lower. As a result, a high-pitched sound corresponds to an increase of the heart rate, and a low frequency sound to a decrease. This basically reduces the details to a binary information $\operatorname{sign}\left(t_{\text {actual }}-t_{\text {expected }}\right)$. A more continuous variant could be called Chirped Pitch where the pitch deviation is set proportional to $t_{\text {actual }}-t_{\text {expected }}$, so that the size of pitch deviations becomes a continuous cue that informs about the amount of temporal mismatch. 


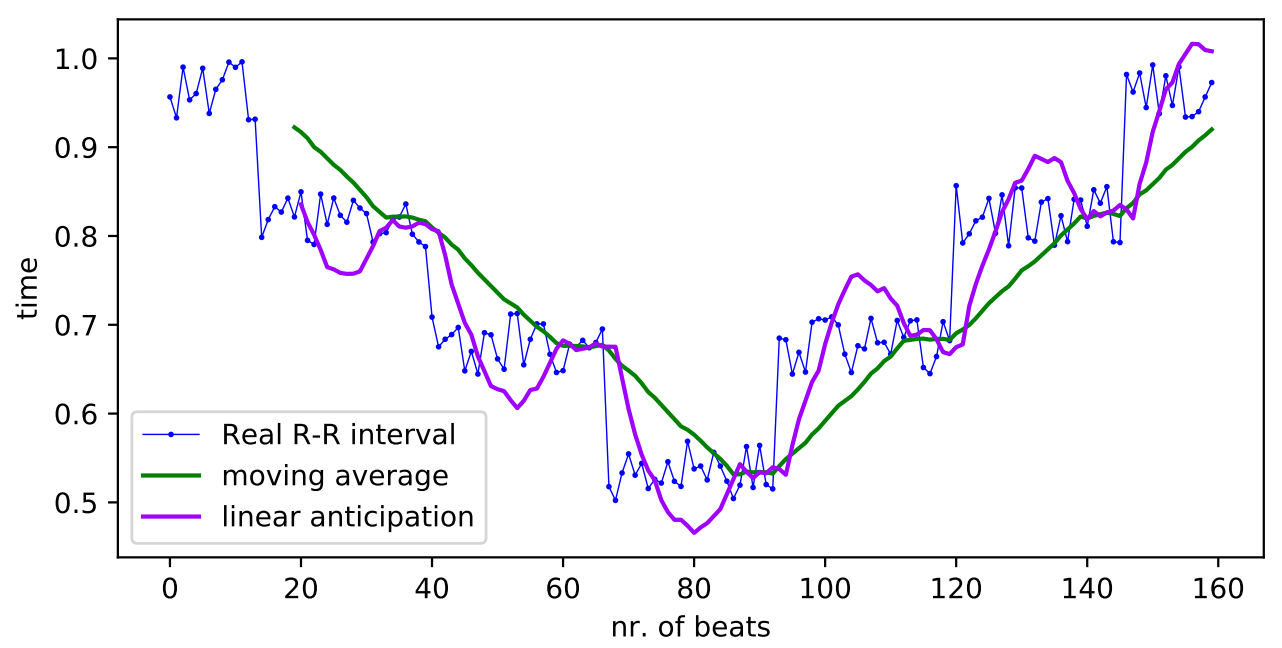

Figure 4: Computation of the anticipation signal on surrogate data: (i) Real RR interval, (ii) Expected RR interval using averaging, (iii) Expected RR interval using linear regression.

Sonification example $\mathrm{S}^{7}$ illustrates the Polarity-Pitch sonification for a dataset classified as healthy, while S2, S3 and S4, present the sonification for datasets containing Atrial fibrillation, Atrial flutter and Ventricular Fibrillation respectively.

\subsubsection{ECGrains sonification}

In noisy environments, such as an emergency room, perceived changes in duration or pitch from the previous method could not be enough to detect subtle variations. Also, there are other ECG features that can be taken into account when sonifying arrhythmias. For instance, as explained in section 2, pathologies such as atrial flutter and atrial fibrillation present a change in the morphology of the P-wave. In positive ECG leads (current flowing towards the positive electrode), atrial flutter presents a $2: 1,3: 1$ or $4: 1$ ventricular rate, meaning that during each heart beat there could be two, three or up to four P-waves present per QRS complex. Also, in atrial fibrillation the P-wave is absent. Therefore, in this sonification we look into other mappings that contribute to make arrhythmia features more salient and besides using the anticipated signal, we also consider if the P-wave is present in the signal and which is its duration.

In the ECGrains sonification we use a granular synthesis generator. Granular synthesis is based on using very short fragments of sound (called grains) that can be controlled and modified to form a new output. We can control parameters such as the number of fragments to be triggered, the duration of each fragment, among others. In our sonification the grains are sine waves, however any sound could be fragmented in the same way to provide a wide palette of synthesized sounds.

We propose a parameter-mapping sonification where we trigger a granulated sound whenever an $\mathrm{R}$ peak is found. The number of small fragments (grains) that compose the synthesized sound is determined based on the difference between the expected peak and the actual peak (anticipation signal). As a result, the lower the difference between actual and expected $\mathrm{R}$ peaks, the lower the

\footnotetext{
${ }^{7}$ See section 7 for suplementary material.
}

number of grains ( 1 grain is the minimum amount to be triggered with each $\mathrm{R}$ peak). This means that a healthy ECG signal, with no abrupt variations between actual versus expected onset, would produce a granulated signal containing only one grain whenever an $\mathrm{R}$ peak is found. However, a pathological signal would create a completely different rhythmical pattern because the resulting sound would contain more grains. The duration of the granulated sound event in each $\mathrm{R}$ peak, is bound to the duration of the last RR interval, regardless of the number of grains in it.

In order to estimate the presence and duration of the P-wave, we calculate the average amplitude in each heart beat and determine how many data points are above a given threshold during the part where the $\mathrm{P}$-wave is expected to appear. We take as a reference Fig. 1 where in a healthy dataset we expect a positive P-wave marking the beginning of the ECG cycle.

The expected location and duration of the $\mathrm{P}$-wave is predicted taking into consideration the typical ECG waves' duration for a heart rate of 60 Beats Per Minute (bpm) ${ }^{8}$ [9], however, in our calculations, we estimate these values based on the current heart rate $H R=\frac{60}{\Delta t_{R R}}$. Thus, we count how many data points exceed a given threshold within the previously defined "P-wave search" area, and use the resulting number as an ECG feature which we map to the frequency value of the resulting sound.

In the mapping, we set the range (min, max) of the source value (P-wave duration) to (0.0, expected duration of the P-wave according to heart rate) and the output ( $\min , \max$ ) is set to a pitch corresponding to MIDI notes ranging from $(80,62)$. This means that an absent or uncommon P-wave morphology, in which the number of data points above the P-wave threshold is low or inexistent, will produce a high pitched sound. On the contrary, a healthy dataset produces a low pitched sound because the number of data points exceeding the threshold is large.

The motivation for selecting low frequencies values to represent healthy states and high frequencies for pathological states, is that in healthy conditions we want the resulting sound to be simi-

${ }^{8} \mathrm{P}$-wave width normal value $=100 \mathrm{~ms}(60 \mathrm{bpm})$ 
lar to the heart heard through a stethoscope, however, if anything abnormal happens in the signal, we want to increase the sound frequency to call attention from the listener.

Sonification example S5 corresponds to the ECGrains sonification of a healthy dataset, while S6, S7, and S8 correspond to datasets with Atrial Fibrillation, Atrial Flutter and Ventricular Fibrillation respectively.

\subsubsection{Marimba sonification}

The Marimba sonification is based on the ECGrains sonification, however, instead of using sine waves as the grains in the synthesizer, we use audio samples of the musical instrument known as marimba. The motivation to do this is that at present, the sonifications used in clinical environments are mainly based on sine oscillators, but at the same time, clinicians often report that these sounds produce auditory fatigue. For this reason, we want to explore how other sounds are perceived in the same environment and if they could be used for long hours of monitoring a signal. In particular, we chose the marimba because it is a percussive sound with an envelope similar to the sound produced by the heart when heard through a stethoscope.

For this sonification, the number of small grains that compose the synthesized sound depend on the time difference between the expected peak and the actual peak that we calculate using the anticipation signal method. As a result, a dataset where there are not important rhythm disturbances, will produce a sonification that triggers one marimba sound when an $\mathrm{R}$ peak is detected. On the contrary, if the difference between actual and expected $\mathrm{R}$ peaks is higher, a larger number of grains will be triggered in each heartbeat. The frequency of the granulated sound in each heart cycle is set to either a $\mathrm{C}$ note or an $\mathrm{E}$ note. These two notes are alternated throughout the sonification.

Sonification example S9 corresponds to the Marimba sonification of a healthy dataset, while S10, S11, and S12 correspond to datasets with Atrial Fibrillation, Atrial Flutter and Ventricular Fibrillation respectively.

\section{PRELIMINARY PERCEPTION TEST}

We conducted a preliminary perception test involving six clinicians (one experienced cardiologist, one cardiologist in training, 1 anesthetist, 2 emergency physicians, and one nurse of intensive care medicine). The participants were asked to rate on a 7-point Likert scale, where 1 means Not at all and 7 means Very much, their agreement to five statements. The evaluated features were: (a) if the sonification was pleasant to listen to, (b) the suitability of the sonification to be heard for a longer period of time during a cardiac procedure or when working in the operation theater, (c) suitability of the sonification for everyday use in the medical emergency scenario: listening to it in the emergency room or emergency vehicle, (d) if the patients would not be annoyed, frightened or intimidated by the sound (e) if the sonification is informative (it gives an idea about the nature of the ECG data between healthy and arrhythmic states). At the end of the test the participants could add any comments they considered pertinent.

Fig. 5, shows the results of the preliminary perceptions test. Results are reported as $(\mu \pm \sigma)$ in a scale from 1 to 7 ( 1 refers to Not at all and 7 to Very much). Pleasantness of the sonification, evaluated in question A, was rated $3.66 \pm 1.36$ for the PolarityPitch sonification, $4.83 \pm 1.72$ for the ECGrains sonification and

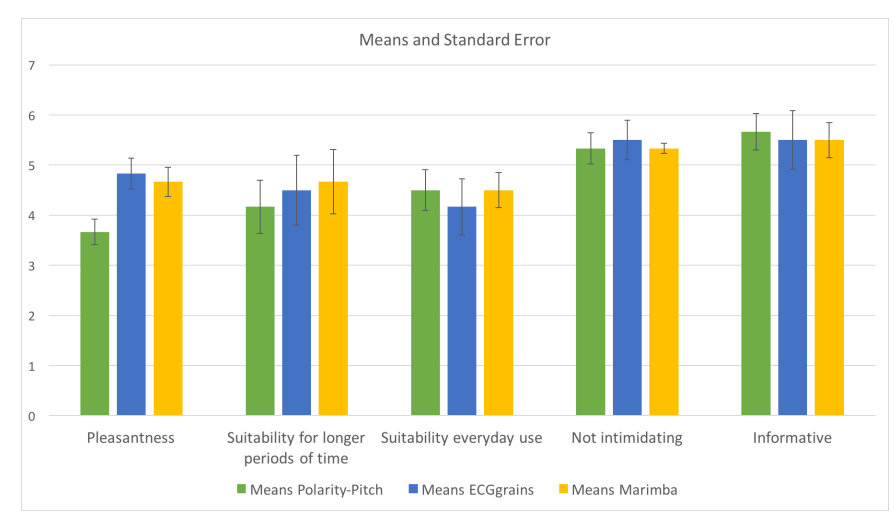

Figure 5: Results of the preliminary perception test (Means and Standard Error bars).

$4.66 \pm 1.03$ for the Marimba sonification. Question 5, which evaluated if the information was informative was rated as $5.66 \pm 1.86$ for the Polarity-Pitch sonification, $5.5 \pm 1.87$ for the ECGrains sonification and $5.5 \pm 1.37$ for the Marimba sonification.

According to the results, the participants found the sonifications to be informative, which means that the sonifications could help to assess rhythm disturbances in the ECG. However, there is no agreement regarding the most appropriate sonification type. In all the aspects consulted there is a large variance, even though all participants are related to the medical field, their interaction with ECG data occurs in different environments (e.g., doctor's office, emergency room, operation theater) and therefore they could have diverse expectations about the sonifications. Nevertheless, regarding the sonification designs, the Marimba sonification presents the lowest variance across all questions, indicating that the participants agreed more about the rating of this sound.

Not all the participants included comments at the end of the test, but one of them mentioned that with the Polarity-Pitch sonification and the Marimba sonification it was easier to listen to the ECG information than with the ECGrains sonification. Another user commented that the sonifications draw too much attention, which could be interpreted as distracting.

After the preliminary perception test, we plan to conduct a study that evaluates ECG classification accuracy (e.g., healthy and pathological states) and assesses benefits in reducing just noticeable differences (JND) for detecting rhythm disturbances when compared under three feedback conditions (visual, auditory and visual+auditory).

\section{DISCUSSION AND CONCLUSIONS}

This work presents a system to acquire, process and sonify ECG signals in real-time. The system is aimed to assist users to monitor their heart without distractions from a visual display. In this paper we focused on the diagnostic and monitoring of arrhythmias, however the system and the sonification methods can be extended to other cardiac pathologies.

CardioSounds can be used in clinical environments to assist clinicians to monitor their patients' biosignals while focusing on a primary task such as performing a cardiac procedure, examination of the patient or revising their medical history. It can also be used by people interested in monitoring their heart while do- 
ing sports, especially when there is evidence of previous abnormal heart activity. For instance, persons who suffered a myocardial infarction could present post MI arrhythmias that depending on their characteristics could become life-threatening rhythm disturbances. For this reason, it is important to monitor the appearance of these pathologies.

The current system setup supports the recording and sonification of one ECG lead. However, it is possible to have multivariate extensions by adding more ECG modules with their respective sensors to the BITalino platform. We consider important to include the recording of at least another lead to have a better overview of the heart's state. Also, given that the analysis process runs on a Raspberry Pi device, for the moment, visualization of the data relies on an external display, yet we plan to include this option as part of the CardioSounds app. So far the mobile application includes basic control over the system but it is in our plans to increase the number of commands that the user can use to interact with it. For example, graphical elements for controlling the sonification's parameters, playback rate, among others, are yet to be added to the application.

Regarding the sonifications, we propose three sonification designs that intend to magnify even subtle changes in consecutive RR intervals. The proposed methods are a continuation of our previously presented sonification methods [1] to magnify subtle differences in rhythm. In our current sonification design, we account for a wider range of parameters in the ECG signal and map them to a broader set of auditory parameters. The sonifications presented are based on the Anticipatory Mapping method where we compare the time difference between the actual onset of the detected $\mathrm{R}$ peak to the expected onset. We calculate the expected onset using forecasting methods such as averaging and linear regression. In this paper we present a basic approach to sonify changes in the P-wave morphology, however, future sonifications should rely on a better estimation method for the $\mathrm{P}$-wave duration.

The preliminary qualitative perception test showed that the sonifications are considered informative when it comes to detecting rhythm disturbances in the ECG signal, however the opinions about the preferred sonification type or the suitability for everyday use in the medical context differ greatly from subject to subject. Given that all the participants work with ECG data in different scenarios (e.g., doctor's office, emergency room), it could be that the expectations they have about how the sonifications should sound or the information they should convey, differs. Nonetheless, in the upcoming study we need to include a larger sample size in order to reduce variance.

In the next stage of development of CardioSounds, we plan to extend the capabilities of the mobile application so that users can store and retrieve ECG recordings. This would allow users to modify the temporal resolution of the retrieved sonifications to listen to them at a faster or slower pace in order to do a quick scan of the data or focus on a specific part of it. We also intend to conduct a study where clinicians and non-clinician listeners can evaluate if the sonification contributes to the detection of heart rhythm disturbances when compared to traditional visual monitoring methods.

\section{RESOURCES}

Examples of the sonifications are provided in: http://dx.doi.org/10.4119/unibi/2919694

\section{ACKNOWLEDGMENT}

This work has been supported by the German Academic Research Service (DAAD) and the Cluster of Excellence Cognitive Interaction Technology "CITEC" (EXC 277) at Bielefeld University, which is funded by the German Research Foundation (DFG).

\section{REFERENCES}

[1] A. L. A. Blanco, S. Grautoff, and T. Hermann, "Cardiosounds: Real-time auditory assistance for supporting cardiac diagnostic and monitoring," in Proceedings of the 12th International Audio Mostly Conference on Augmented and Participatory Sound and Music Experiences, ser. AM '17. New York, NY, USA: ACM, 2017, pp. 45:1-45:4. [Online]. Available: http://doi.acm.org/10.1145/3123514.3123542

[2] D. Chandra, M. J. Tessler, and J. Usher, "Audio spectrum and sound pressure levels vary between pulse oximeters," Canadian Journal of Anesthesia, vol. 53, no. 1, pp. 26-32, Jan 2006. [Online]. Available: https: //doi.org/10.1007/BF03021524

[3] H. Terasawa, Y. Morimoto, M. Matsubara, A. Sato, M. Ohara, and M. Kawarasaki, "Guiding auditory attention toward the subtle components in electrocardiography sonification," in Proc. ICAD. Georgia Institute of Technology, 2015.

[4] J. N. Kather, T. Hermann, Y. Bukschat, T. Kramer, L. R. Schad, and F. G. Zllner, "Polyphonic sonification of electrocardiography signals for diagnosis of cardiac pathologies," Scientific Reports, vol. 7, p. 44549, 2017.

[5] P. Vickers, "Sonification for process monitoring," in The Sonification Handbook, T. Hermann, A. Hunt, and J. G. Neuhoff, Eds. Berlin, Germany: Logos Publishing House, 2011, ch. 18, pp. 455-491. [Online]. Available: http://sonification.de/handbook/chapters/chapter18/

[6] F. Foxall and H. Blackburn, Cardiac Arrhythmia Recognition : An Easy Learning Guide., ser. Easy Learning Guides From M and K. M and K Update Ltd, 2010. [Online]. Available: http://search.ebscohost.com/login.aspx?direct= true $\& d b=$ nlebk\&AN=400965\&site=ehost-live

[7] M. G. Tsipouras, D. I. Fotiadis, and D. Sideris, "Arrhythmia classification using the rr-interval duration signal," in Computers in Cardiology, Sept 2002, pp. 485-488.

[8] J. Hirsch and B. Bishop, "Respiratory sinus arrhythmia in humans: How breathing pattern modulates heart rate," vol. 241, pp. H620-9, 111981.

[9] G. D. Clifford, F. Azuaje, and P. Mcsharry, "Ecg statistics, noise, artifacts, and missing data," Advanced Methods and Tools for ECG Data Analysis, vol. 6, p. 18, 2006.

[10] D. Worrall, B. Thoshkahna, and N. Degara, "Detecting components of an ecg signal for sonification," in Proc. ICAD. Georgia Institute of Technology, 2014.

[11] B. Moore, Psychoacoustics. New York, NY: Springer New York, 2007, pp. 459-501. [Online]. Available: https://doi.org/10.1007/978-0-387-30425-0_13

[12] A. Friberg and J. Sundberg, "Perception of justnoticeable time displacement of a tone presented in a metrical sequence 
at different tempos," The Journal of the Acoustical Society of America, vol. 94, no. 3, pp. 1859-1859, 1993. [Online]. Available: https://doi.org/10.1121/1.407650 